DOI: $10.21767 / 2471-8157.100031$

\title{
Dobutamine Dose: What are its Effects?
}

\author{
Rabindra Nath Das* \\ Department of Statistics, The University of Burdwan, Burdwan, West Bengal, India
}

*Corresponding author: Rabindra Nath Das, Department of Statistics, The University of Burdwan, Burdwan, West Bengal, India, Tel: 342263 4975; E-mail: rabin.bwn@gmail.com

Rec Date: Aug 17, 2016, Acc Date: Oct 18, 2016, Pub Date: Oct 22, 2016

Citation: Das RN. Dobutamine Dose: What are its Effects? Interv Cardiol J 2016, 2:3.

Abstract

Dobutamine stress echocardiography (DSE) is successfully and widely used to identify whether a patient with or without known coronary artery disease has ischemia. This article focuses the effects of Dobutamine dose (DD) who underwent DSE, based on the data set in UCLA Statistics Web Site. The analysis results of DD, and the effects of DD on different blood pressures, cardiac ejection fractions, and heart rates are discussed for the awareness of the healthy individuals, cardiac patients, and medical practitioners.

Keywords: Blood pressure; Cardiac ejection fraction; Coronary heart disease; Dobutamine dose; Gamma model; Heart rate; Non-constant variance

\section{Dobutamine Dose Effects}

DSE continues to be successfully used to identify whether the patients with and without known coronary artery disease have ischemia [1-4]. In practice, DSE is applied if the patient is unable to exercise by treadmill or bicycle to a certain degree that will provide useful clinical information. DSE is used to obtain adequate imaging at all levels of the patient's stress without his/her cooperation. Due to DSE used, echocardiographer has enough time to take the necessary imaging of the stress at all levels. It is well known that the blood pressures, cardiac ejection fraction, and the heart rate are highly associated with the cardiovascular disease. Blood pressures and hypertension are highly associated, and hypertension is associated with stroke for $54 \%$, and $47 \%$ of ischaemic heart disease [5], and it affects $30 \%$ of the adult population [6]. Ejection fraction (EF) and the heart rates are related with the severity of the systolic heart failure [7]. Only the advantages of DSE used are known in the literature. To the best of our knowledge, effects of DSE used are little known in the literature.

We seek answers to the following questions: What are the effects of Dobutamine dose (DD) on the cardiac patients who underwent DSE? Does DD effect on different blood pressures, cardiac ejection fractions, and heart rate? These answers are derived based on the data set of 31 variables on 558 subjects (UCLA stress echocardiography data). A detailed description of the data set, collection method, patient population, and the DSE is given in [8] ref. The variables/factors of this study are basal heart rate (bpm) (coded as bhr), basal blood pressure (mmHg) (coded as basebp), basal double product (DP) (bhr*basebp) (bpm*mmHg) (coded as basedp), peak heart rate (bpm) (coded as pkhr), systolic blood pressure $(\mathrm{mmHg})$ (coded as sbp), DP pkhr*sbp (bpm*mmHg) (coded as dp), Dobutamine dose (DD) given (coded as dose), maximum heart rate (bpm) (coded as maxhr), percent maximum predicted heart rate (coded as pctMphr), maximum blood pressure $(\mathrm{mmHg})$ (coded as mbp), DP on max DD (bpm*mmHg) (coded as dpmaxdo), DD at max double product (mg) (coded as dobdose), age (years) (coded as age), gender (male=0, female $=1$ ) (coded as gender), Baseline cardiac ejection fraction (coded as baseEF), ejection fraction on Dobutamine (coded as dobEF), chest pain (yes $(y)=0$, no $(n)=1)$ (coded as chest pain), resting wall motion abnormality on echocardiogram (ECDG) $(y=0, n=1)$ (coded as restwma), positive stress ECDG $(y=0, n=1)$ (coded as posSE), new myocardial infraction (MI) $(y=0, n=1)$ (coded as newMI), recent angioplasty $(y=0, n=1)$ (coded as newPTCA), recent bypass surgery $(y=0, n=1)$ (coded as newCABG), death $(y=0, n=1)$ (coded as death), history of hypertension $(y=0, n=1)$ (coded as hxofHT), History of diabetes $(\mathrm{y}=0, \mathrm{n}=1)$ (coded as hxofDM), history of smoking (nonsmoker $=0$, moderate $=1$, heavy $=2$ ) (coded as hxofCig), history of $\mathrm{MI}(\mathrm{y}=0, \mathrm{n}=1)$ (coded as hxofMI), history of angioplasty $(y=0$, $n=1$ ) (coded as hxofPTCA), history of coronary artery bypass surgery $(y=0, n=1)$ (coded as hxofCABG), death, newMl, newPTCA or newCABG (death $=0$, no $=1$ ) (coded as any event), Baseline electrocardiogram diagnosis (normal=0, equivocal=1, $\mathrm{MI}=2$ ) (coded as ECG). This data set has been analyzed by the joint gamma models [9]. The effects of DD on these variables/ factors have been derived by modeling DD on these variables. Also, the different blood pressures, cardiac ejection fractions, and the heart rates are separately modeled with the remaining other variables/factors, along with the DD to identify the effects of DD. The effects of DD on the cardiac patients who underwent DSE are described as follows.

By modeling DD on the remaining other variables/factors, the following effects of DD can be obtained. The mean DD is negatively associated with the double product (DP) of pkhr and sbp (dp) $(P<0.001)$. If DD is high, dp will be low. Mean DD is separately positively associated with the dpmaxdo $(P<0.001)$ and dobdose $(P<0.001)$. If $D D$ is high, both dpmaxdo and dobdose are also high. Practically, DD has direct association separately with both the dpmaxdo and dobdose. DD variance is separately negatively associated with sbp $(P<0.001)$, dobdose $(P<0.001)$, gender $(P=0.019)$, newMI $(P<0.001)$, death $(P=0.073)$. Therefore, the DD variance is high at low sbp or low 
dobdose. The DD variance is also high for the cardiac patients with male sex or newMl or who are close to death. The DD variance is separately positively associated with $\mathrm{mbp}$ $(P<0.001)$, posSE $(P=0.011)$, hxofMI $(P=0.096)$. So, the $D D$ variance is high at high $\mathrm{mbp}$. Also, the $\mathrm{DD}$ variance is high for the cardiac patients with no posSE or hxofMI. Note that in epidemiology, partially significant factors are known as confounders.

By modeling separately basal blood pressure (basebp), systolic blood pressure (sbp), and maximum blood pressure (mbp) on the remaining other variables/factors, the following effects of DD can be obtained. The mean basebp is negatively associated with the dpmaxdo $(P<0.001)$. The basebp is low at high dpmaxdo. The variance of basebp is positively associated with the dpmaxdo $(\mathrm{P}<0.001)$. So, at high dpmaxdo, the basebp variance is also high. The mean sbp is positively associated with the dose $(P=0.032)$, and it is negatively associated with the dpmaxdo $(P<0.001)$. So, the sbp is high, at high dose, and at low dpmaxdo. The mean mbp is positively associated with the dpmaxdo $(\mathrm{P}<0.001)$. It indicates, $\mathrm{mbp}$ is high at high dpmaxdo.

By modeling separately baseline cardiac ejection fraction (baseEF), and ejection fraction on Dobutamine dose (dobEF) on the remaining other variables/factors, the following effects of DD can be obtained. The mean baseEF is negatively associated with the dose $(P=0.025)$, indicating that the baseEF is low at high dose. Again, the mean dobEF is positively associated with the dose $(P=0.011)$, indicating that bobEF is high at high dose. Also, the dobEF variance is negatively associated with the dobdose $(P=0.001)$, indicating that the dobEF variance is high at low dobdose.

By modeling separately basal heart rate (bhr), peak heart rate (pkhr), and maximum heart rate (maxhr) on the remaining other variables/factors, the following effects of DD can be obtained. The mean bhr is separately negatively associated with the dpmaxdo $(P<0.001)$ and dobdose $(P=0.074)$. Thus, bhr is low at high dpmaxdo or dobdose. The mean pkhr is positively associated with the dpmaxdo $(P<0.001)$, and the pkhr variance is negatively associated with the dobdose $(\mathrm{P}<0.001)$. This implies that the mean pkhr is high at high dpmaxdo, and the pkhr variance is high at low dobdose. The mean maxhr is positively associated with the dpmaxdo $(P<0.001)$, and the maxhr variance is positively associated with the dobdose $(P<0.001)$. So, the maxhr is high at high dpmaxdo, and maxhr variance is high at high dobdose.

The above summarized results are given in Table 1, and these are derived based on joint gamma models [9]. Here only the important mean and the variance parameters of the responses are very shortly discussed. All the derivations along with many mean and dispersion parameters will be discussed in the full research papers. The complete research papers will be submitted very soon. The above mentioned Dobutamine dose effects are associated with DSE patients. It is observed that the DD and its interaction effects dpmaxdo and dobdose have many significant effects. This report recommends the following for all individuals. Medical practitioners should be care on Dobutamine dose, and its interaction effects with other risk factors. Due to DD, blood pressures, heart rates and cardiac ejection fraction have been effected. Due to high DD, sbp is high, and baseEF is low, consequently stroke may be associated with DD. If possible, try to avoid DD. To apply DD, medical practitioners should care on the patient's cardiac significant variables/factors as pointed above.

Table 1: Association of Dobutamine dose with different factors.

\begin{tabular}{|c|c|c|c|}
\hline Response & Associated with & $\begin{array}{l}\text { Association } \\
\text { type }\end{array}$ & P-value \\
\hline \multirow[t]{3}{*}{ Mean of DD } & $\mathrm{Dp}$ & negative & $P<0.001$ \\
\hline & dpmaxdo & Positive & $P<0.001$ \\
\hline & dobdose & Positive & $P<0.001$ \\
\hline \multirow[t]{8}{*}{ Variance of DD } & Sbp & negative & $P<0.001$ \\
\hline & dobdose & negative & $P<0.001$ \\
\hline & Gender & negative & $P=0.019$ \\
\hline & newMl & negative & $P<0.001$ \\
\hline & Death & negative & $P=0.073$ \\
\hline & Mbp & Positive & $P<0.001$ \\
\hline & posSE & Positive & $P=0.011$ \\
\hline & hxofMl & Positive & $P=0.096$ \\
\hline Mean of basebp & dpmaxdo & negative & $P<0.001$ \\
\hline $\begin{array}{l}\text { Variance of } \\
\text { basebp }\end{array}$ & dpmaxdo & Positive & $P<0.001$ \\
\hline \multirow[t]{2}{*}{ Mean of sbp } & Dose & Positive & $\mathrm{P}=.032$ \\
\hline & dpmaxdo & negative & $P<0.001$ \\
\hline Mean of mbp & dpmaxdo & Positive & $P<0.001$ \\
\hline Mean of baseEF & Dose & negative & $P=0.025$ \\
\hline Mean of dobEF & Dose & Positive & $P=0.011$ \\
\hline $\begin{array}{l}\text { Variance of } \\
\text { dobEF }\end{array}$ & dobdose & negative & $P=0.001$ \\
\hline \multirow[t]{2}{*}{ Mean of bhr } & dpmaxdo & negative & $P<0.001$ \\
\hline & dobdose & negative & $P=0.074$ \\
\hline Mean of pkhr & dpmaxdo & Positive & $P<0.001$ \\
\hline Variance of pkhr & dobdose & negative & $P<0.001$ \\
\hline Mean of maxhr & dpmaxdo & positive & $P<0.001$ \\
\hline Variance of maxhr & dobdose & positive & $P<0.001$ \\
\hline
\end{tabular}

\section{References}

1. Marcovitz PA, Armstrong WF (1992) Accuracy of dobutamine stress echocardiography in detecting coronary artery disease. Am J Cardiol 69: 1269-1273.

2. Davila Roman VG, Waggoner AD, Sicard GA, Geltman EM, Schechtman KB, et al. (1993) Dobutamine stress echocardiography predicts surgical outcome in patients with an 
aortic aneurysm and peripheral vascular disease. J Am Coll Cardiol 21: 957-963.

3. Secknus MA, Marwick TH (1997) Evolution of dobutamine echocardiography protocols and indications: safety and side effects in 3,011 studies over 5 years. J Am Coll Cardiol 29: 1234-1240.

4. Bach DS, Hepner A, Marcovitz PA, Armstrong WF (1993) Dobutamine stress echocardiography: prevalence of a nonischemic response in a low-risk population. Am. Heart J 125: 1257-1261.

5. Micha R, Wallace SK, Mozaff arian D (2010) Red and processed meat consumption and risk of incident coronary heart disease, stroke, and diabetes mellitus: a systematic review and metaanalysis. Circulation 121: 2271-2283.
6. Kearney PM, Whelton M, Reynolds K, Muntner P, Whelton PK, et al. (2005) Global burden of hypertension: analysis of worldwide data. Lancet 365: 217-223.

7. Kumar V, Abbas, AK, Aster J (2009) Robbins Cotran pathologic basis of disease (8thedn), St. Louis, Mo Elsevier Saunders, USA.

8. Janine K, Child Jhon S, Garfinkel WDO, Alan (1999) Prognostic value of dobutamine stress echocardiography in predicting cardiac events in patients with known or suspected coronary artery disease. J Am Coll Cardiol 33: 708-716.

9. Das RN (2014) Robust Response Surfaces, Regression, and Positive Data Analyses. Chapman \& Hall, London, UK. 